\title{
Systematic Waveform Engineering Enabling High Efficiency Modes of Operation in Si LDMOS at both L-band and S-band Frequencies
}

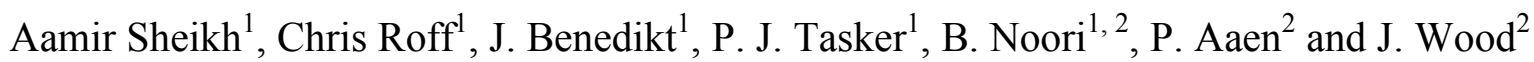 \\ ${ }^{1}$ School of Engineering, Cardiff University, Cardiff, CF23 3AA, U.K (e-mail: sheikha1@cf.ac.uk ) \\ ${ }^{2}$ Freescale Semiconductor, Tempe AZ, USA
}

\begin{abstract}
This paper demonstrates that by robust waveform engineering it is possible for high power Si LDMOS to achieve very high efficiency at frequencies up to $2.1 \mathrm{GHz}$. Class $\mathrm{F}$ amplifier operation was realized in a $5 \mathrm{~W}$ LDMOS device by the successful application of robust waveform engineering procedures; undertaken at the current generator plane. The peak power added efficiency was found to be $78 \%$ at $0.9 \mathrm{GHz}$ and $77 \%$ at $2.1 \mathrm{GHz}$. In both cases the RF waveforms were optimized in terms of the gate voltage, fundamental and harmonic impedances. The main difference at $2.1 \mathrm{GHz}$ was the change in fundamental impedance to a more reactive impedance to compensate for the dynamic device output capacitance. To the authors' knowledge this is the highest efficiencies reported in the literature for $\mathrm{Si}$ LDMOS devices at 2.1GHz.
\end{abstract}

Index Terms - High power, MOSFETs, power amplifiers.

\section{INTRODUCTION}

In the current climate of energy conservation, higher running costs and limited energy supplies there is a great need to produce highly efficient power amplifiers. This is especially applicable for high power applications such as radar or mobile phone basestations where the amplifiers are typically outputting in excess of $40 \mathrm{dBm}$.

One such high efficiency implementation is the Class $\mathrm{F}$ amplifier that allows for a maximum efficiency of $100 \%$ but also allows for maximum output power. The optimal design of a class $\mathrm{F}$ amplifier is done through waveform engineering at the device output [1] and this has been successfully achieved at low power levels in an on wafer environment [2] - [3].

However, the use of waveform engineering in high power devices requires the consideration of extra factors which can significantly alter the waveforms [4]. These are the packaging of the device and the measurement system impedance. Once these affects are negated, waveform engineering can again be applied. In this paper the successful use of waveform engineering has been demonstrated on a $5 \mathrm{~W}$ Si LDMOS device supplied by Freescale Semiconductor. The resulting robust waveform engineering was undertaken at both $0.9 \mathrm{GHz}$ and $2.1 \mathrm{GHz}$ using the high power active harmonic load pull measurement system developed at Cardiff University [5].

There is a large amount of literature reporting high efficiency LDMOS designs with power added efficiencies above $75 \%$ [6]. However, this has only been achieved at frequencies around $1.2 \mathrm{GHz}$. At frequencies approaching Sband the efficiencies typically reported are a lot lower and in the range of $60-65 \%$ [6] which is effectively no better than a class B amplifier. This paper outlines how a proven high efficiency design process [1] can be applied to the latest $\mathrm{Si}$ LDMOS devices at S-band frequencies to achieve efficiencies close to theoretical class-F operation under band limited conditions.

\section{De-EMBedDing to the CuRREnt Generator Plane}

A key requirement for waveform engineering is for procedures to be applied at the current generator plane. Only at this plane is there a direct correlation of the waveforms to the DCIV plane and this also allows for extraction of the device output capacitance which needs to be resonated out if an open circuit is to be presented at the current generator plane. This was done by de-embedding the measured I-V waveforms to the current generator plane. This required the subtraction of the displacement currents and voltages generated by the device and package parasitic components [4].

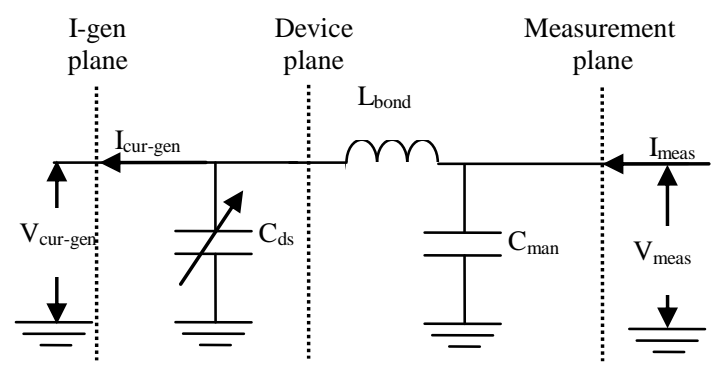

Fig. 1 Device and package parasitic circuit model

The package and device model, which was employed for RF waveform de-embedding, is shown in Fig. 1, and consists of a manifold/bond pad capacitance, bonding wire inductance and a non linear device output capacitance [7]. The package model allowed for the calculation of impedances required at the measurement plane to present the desired impedances at the current generator plane as shown in Table I. A linear capacitance was used in an s-parameter simulation, thus allowing engineering of class $\mathrm{F}$ behavior at the current generator plane. 


\section{TABLE I}

SUMMARY OF THE IMPEDANCES REQUIRED AT THE MEASUREMENT PLANE TO PRODUCE SHORT AND OPEN CIRCUIT TERMINATIONS AT THE CURRENT GENERATOR PLANE

\begin{tabular}{|l|l|l|l|l|}
\hline Frequency & $2^{\text {nd }}$ Short & $2^{\text {nd }}$ Open & $3^{\text {nd }}$ Short & $3^{\text {rd }}$ Open \\
\hline $0.9 \mathrm{GHz}$ & $0-6.8 \mathrm{j} \Omega$ & $0+48.7 \mathrm{j} \Omega$ & $0-9.8 \mathrm{j} \Omega$ & $0+31.5 \mathrm{j} \Omega$ \\
\hline $2.1 \mathrm{GHz}$ & $0-20.1 \mathrm{j} \Omega$ & $0+10.1 \mathrm{j} \Omega$ & $0-23 \mathrm{j} \Omega$ & $0+1.2 \mathrm{j} \Omega$ \\
\hline
\end{tabular}

\section{Class F INVESTIGATION}

\section{A. Gate sweeps}

Class $\mathrm{F}$ behavior was achieved through a systematic investigation outlined in [1]. This procedure started with a gate sweep of the device with a real impedance at the fundamental and short circuits at the second and third harmonics. The gate sweep measurement located the appropriate bias condition to null the most significant odd harmonic (i.e. $3^{\text {rd }}$ harmonic) so that it appears only in the voltage waveform, minimizing dissipated energy and thus enhancing efficiency. This was done at $0.9 \mathrm{GHz}$ and $2.1 \mathrm{Ghz}$, the results for $0.9 \mathrm{GHz}$ are shown in Fig. 2.

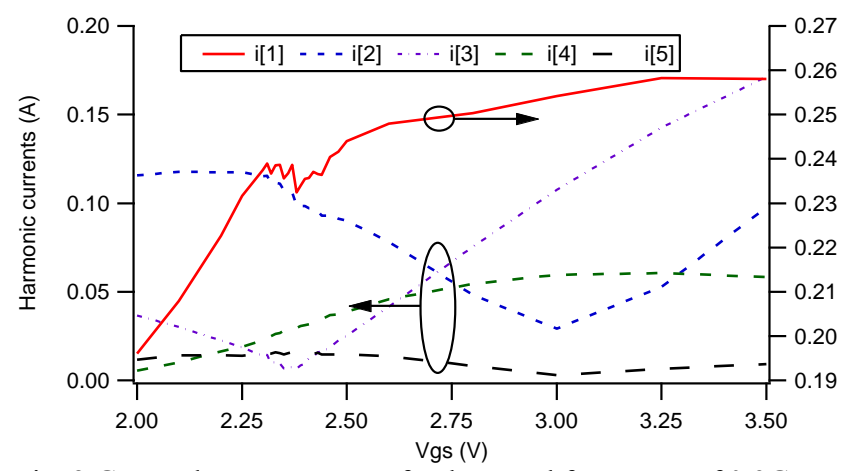

Fig. 2 Gate voltage sweep at a fundamental frequency of $0.9 \mathrm{GHz}$ with the second and third harmonics set to short circuits

The gate sweep at $0.9 \mathrm{GHz}$ shows that there is a clear third harmonic minimum at a gate voltage of $2.37 \mathrm{~V}$, which is slightly above pinch off. At $2.1 \mathrm{GHz}$ the third harmonic minimum does not occur at the same gate voltage. As well as that the third harmonic current minimum is no longer as clearly defined as it was at $0.9 \mathrm{GHz}$. In this case a minimum third harmonic current is observed at a gate voltage of $2.25 \mathrm{~V}$. The reason for this could be due to the relatively low $f_{T}$ which is in the region of $7.5 \mathrm{GHz}$, which starts to affect the harmonic composition of the current waveform when operating at $2.1 \mathrm{GHz}$.

\section{B. Harmonic Optimization}

The harmonic terminations applied at the current generator plane were determined after taking the parasitic network into account. However, due to uncertainties in determining the parasitic components, second and third harmonic phase sweeps, at the two frequencies of operation were conducted, with a constant magnitude set to one. To begin with a second harmonic sweep was done with the third harmonic passively terminated to $50 \Omega$. Once the optimum was determined for the second harmonic a similar phase sweep was done around the third harmonic with the second harmonic now locked to its optimized value. The results are shown in Figs. 3 and 4.

At both frequencies it can be seen that the sensitivity of the second harmonic is very low. This is due to the large device output capacitance, which effectively already provides the required low termination to the current generator. However, in both cases a peak in efficiency is found. At $0.9 \mathrm{GHz}$ this is occurring at $0-14.4 \mathrm{j} \Omega$ and at $2.1 \mathrm{GHz}$ this occurs at $0-24 \mathrm{j} \Omega$. These agree well with the values shown in Table I.

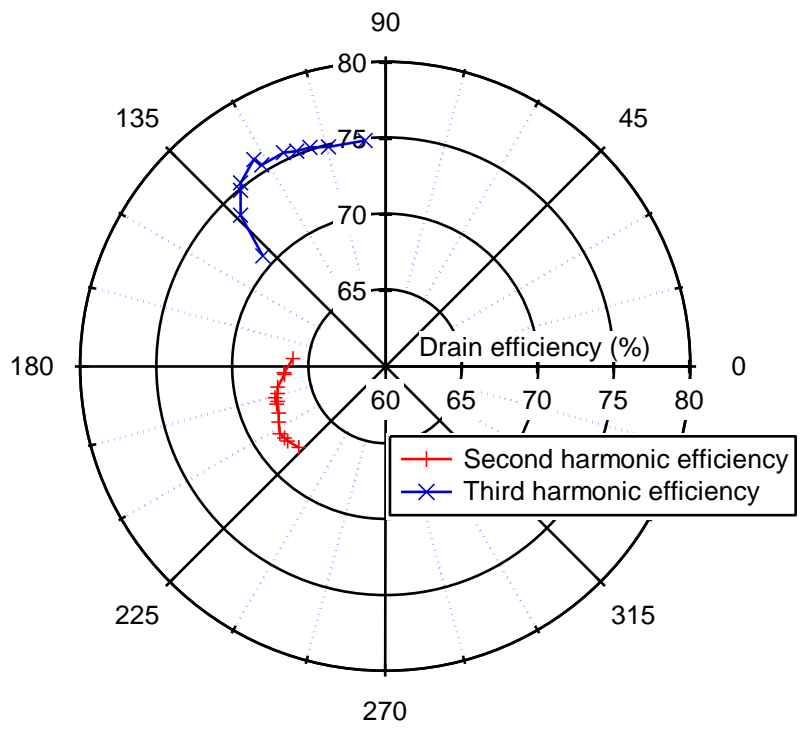

Fig. 3 Phase sweeps at the measurement plane of the second and third harmonic impedances at a fundamental frequency of $0.9 \mathrm{GHz}$

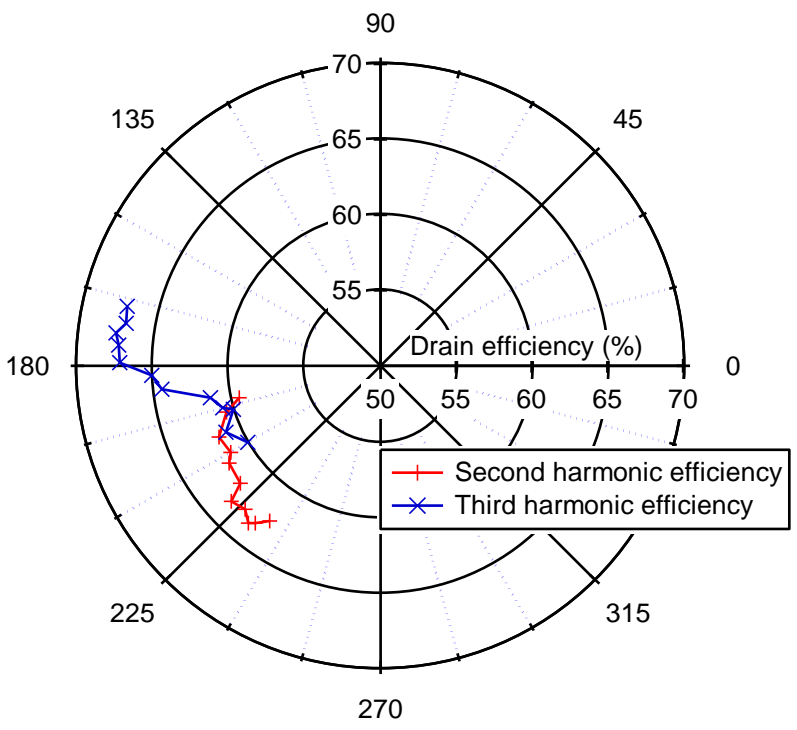

Fig. 4 Phase sweeps at the measurement plane of the second and third harmonic impedances at a fundamental frequency of $2.1 \mathrm{GHz}$ 
The third harmonic phase sweep, used to locate an open, shows a higher level of sensitivity than observed for the second harmonic. This is due to the requirement of resonating out the device output capacitance, which becomes difficult at higher frequencies. This is confirmed by the increased sensitivity of the efficiency at $2.1 \mathrm{GHz}$ where there is a larger variation in efficiency. Peak efficiency at $0.9 \mathrm{GHz}$ is found at $0+28 \mathrm{j} \Omega$ and $0+3.5 \mathrm{j} \Omega$ at $2.1 \mathrm{GHz}$. As before, these are in agreement with the simulated values shown in Table I.

\section{Fundamental Impedance Sweep}

Now that the design has been optimized in terms of its harmonic current and voltage composition, the next step was to enhance the performance of the device at the fundamental. This was done by varying the load presented at the fundamental frequency of operation. The desired real impedance was achieved at the current generator plane by accounting for the package network. This real intrinsic load was then swept over a range of values to determine the optimum fundamental impedance in terms of power added efficiency. Typically, higher drain efficiencies are possible at high fundamental load impedances as the effect of the knee is minimized. However, in this case the power added efficiency (PAE) is used to ensure that the amplifier has sufficient gain. The results are shown in Fig. 5.

The results show that there is an expected decrease in power with load increase but the PAE only noticeably increases up to a fundamental load impedance of $110 \Omega$. For this reason a fundamental load of $110 \Omega$ is chosen as this also provides $36 \mathrm{dBm}(4 \mathrm{~W})$ of output power. This impedance of $110 \Omega$ at the current generator plane was found to be $54.2+52.6 \mathrm{j} \Omega$ at the measurement plane.

At $2.1 \mathrm{GHz}$, the same procedure was applied but it was quickly realized that a real only fundamental impedance was no longer easily achievable. This was due to the effect of the dynamic capacitance, which becomes more prevalent at higher frequencies. The dynamic device output capacitance is a function of the drain voltage [7] and this can not be accounted for using an s-parameter simulation. To minimize the error the 'average' capacitance was determined using the MET [8] model of the device. This was done at the fundamental frequency of operation and this value was inserted into the sparameter simulation. The measured results of the fundamental load sweep at the current generator plane are shown in Fig. 6.

The results in Fig. 6 outline the same performance variation as seen in Fig. 5 but with a lower gain. This is expected due to the relatively low $\mathrm{f}_{\mathrm{T}}$ of the device. This in turn affects the PAE of the device. As can be seen in Fig. 6 the drop in PAE is about three to four percent compared to the results at $0.9 \mathrm{GHz}$.

To confirm that this was the optimum impedance a highdensity fundamental load pull sweep was conducted. The optimum impedance for PAE was found to be $13.9+30.1 \mathrm{j} \Omega$. This was found to be a complex impedance of $99.7+25.9 \mathrm{j} \Omega$ at the current generator plane which is close to the $110 \Omega$ fundamental impedance used at $0.9 \mathrm{GHz}$. The reactive component is possibly due to the complex impedances being presented at the higher harmonics. This occurs due to the $50 \Omega$ system impedance at the higher harmonics being transformed to complex impedances at the current generator plane. Rhodes [9] has theoretically outlined that the optimum impedance for efficiency changes when the higher harmonics are terminated into complex impedances. This new impedance was then used to measure the performance of the device in class F.

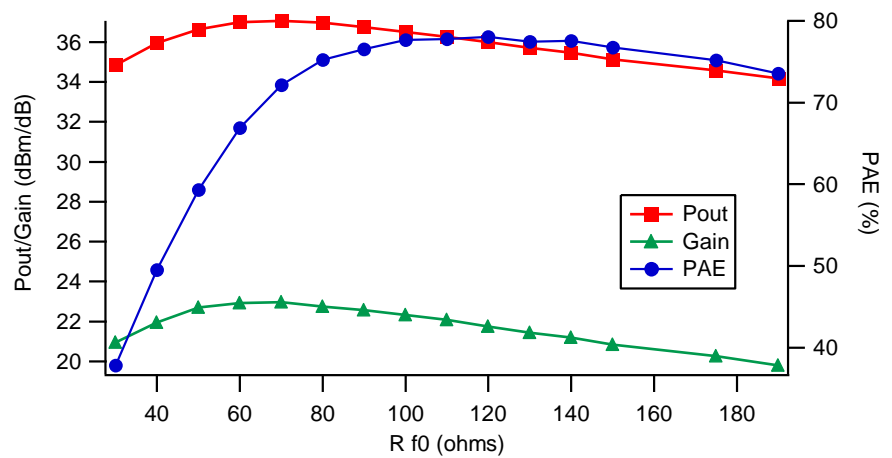

Fig. 5 Fundamental real only load sweep at the current generator plane measured at $0.9 \mathrm{GHz}$

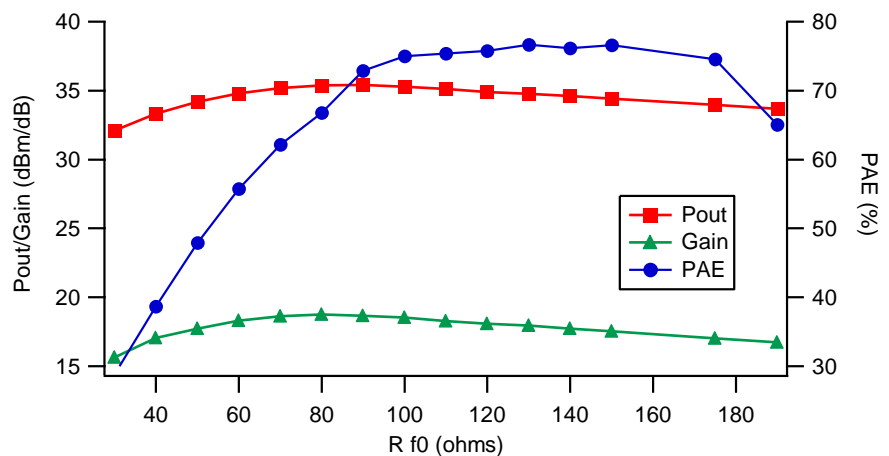

Fig. 6 Fundamental real only load sweep at the current generator plane measured at $2.1 \mathrm{GHz}$

\section{Class F RESUltS}

The device was put into the conditions at the measurement plane as outlined in Table II. The results obtained at $0.9 \mathrm{GHz}$ are shown in Fig. 7. The PAE obtained at $1 \mathrm{~dB}$ compression was $77.2 \%$ whilst delivering $36 \mathrm{dBm}$ of output power to the load. A peak PAE of $78 \%$ was measured with the device $4 \mathrm{~dB}$ into compression.

TABLE II

SUMMARY OF DEVICE SETTINGS FOR CLASS F PERFORMANCE

\begin{tabular}{|l|l|l|l|l|}
\hline Frequency & $\mathrm{Vg}(\mathrm{V})$ & $\mathrm{Z}(\mathrm{f} 0)$ & $\mathrm{Z}(2 \mathrm{f} 0)$ & $\mathrm{Z}(3 \mathrm{f} 0)$ \\
\hline $0.9 \mathrm{GHz}$ & 2.37 & $54.2+52.6 \mathrm{j}$ & $0-14.4 \mathrm{j} \Omega$ & $0+28 \mathrm{j} \Omega$ \\
\hline $2.1 \mathrm{GHz}$ & 2.25 & $13.9+30.1 \mathrm{j}$ & $0-24 \mathrm{j} \Omega$ & $0+3.5 \mathrm{j} \Omega$ \\
\hline
\end{tabular}

The waveforms, at $1 \mathrm{~dB}$ compression are shown in Fig. 8. The waveforms confirm that class $\mathrm{F}$ behavior was obtained at 
the current generator plane. The voltage waveform is shown to be 'squared' in shape and the current is half rectified.

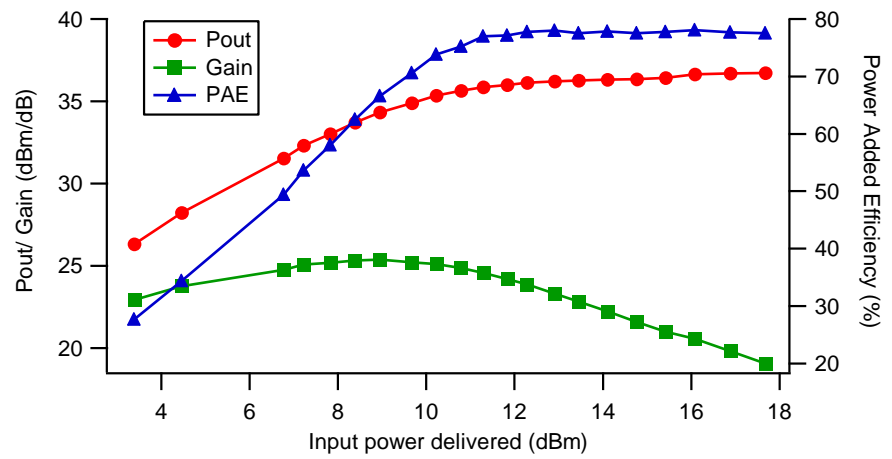

Fig. 7 Performance of the device in class $\mathrm{F}$ at $0.9 \mathrm{GHz}$

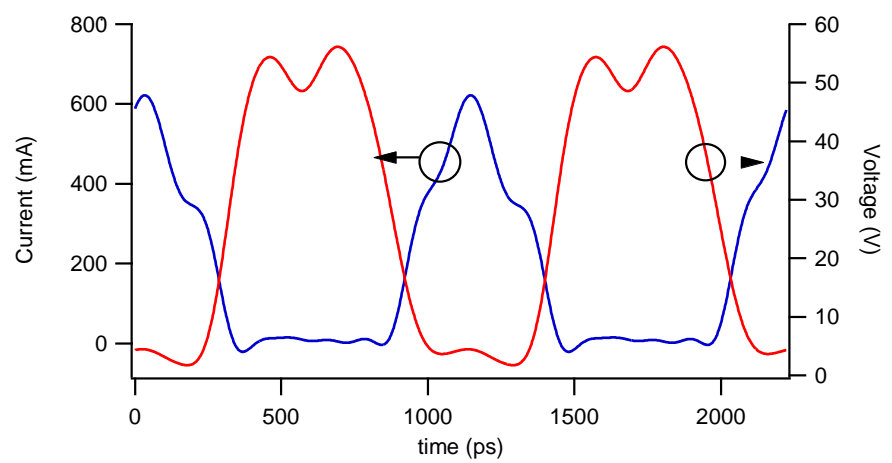

Fig. 8 Measured voltage and current waveforms at the current generator plane at a fundamental frequency of $0.9 \mathrm{GHz}$

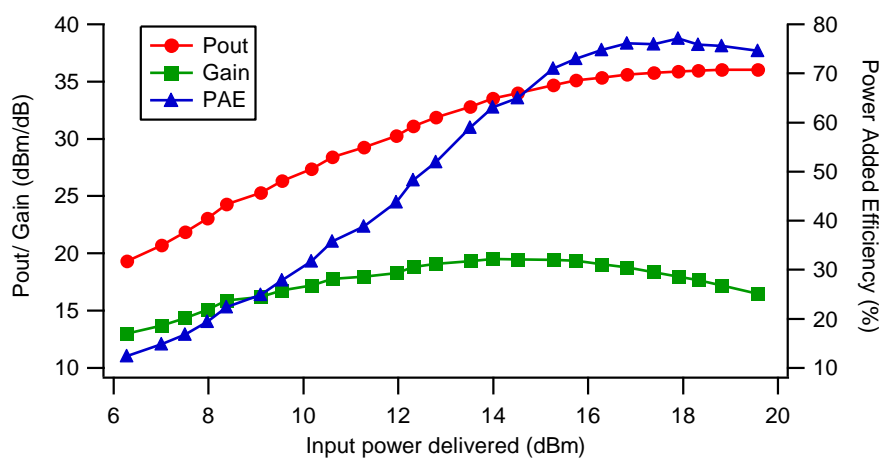

Fig. 9 Performance of the device at $2.1 \mathrm{GHz}$

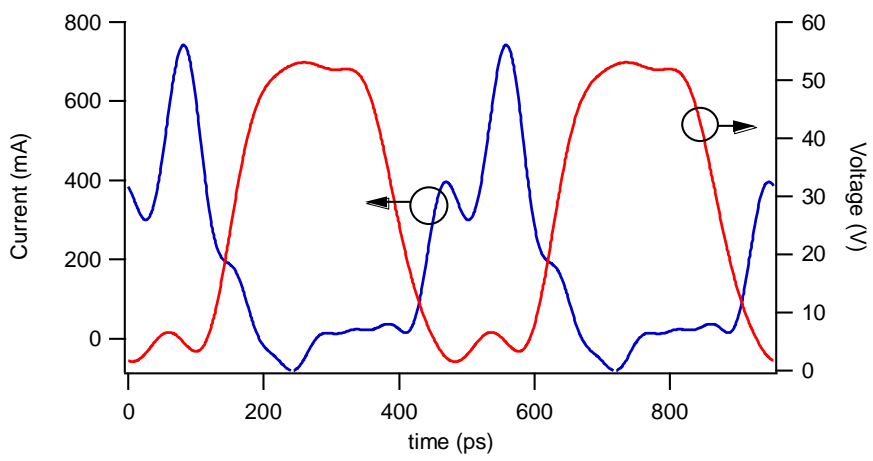

Fig. 10 Measured voltage and current waveforms at the current generator plane at a fundamental frequency of $2.1 \mathrm{GHz}$
The results obtained at $2.1 \mathrm{GHz}$ are shown in Fig. 9. The performance is similar to that obtained at $0.9 \mathrm{GHz}$, with $77.1 \%$ PAE at $1 \mathrm{~dB}$ compression with $35.9 \mathrm{dBm}$ of power being delivered to the load. The main difference is that the flat peak efficiency region found at $0.9 \mathrm{GHz}$ was not replicated at $2.1 \mathrm{GHz}$. The waveforms are shown in Fig. 10.

The voltage waveform is shown to be square in shape as at $0.9 \mathrm{GHz}$ and the current is half rectified. However there is a slight reduction in the third harmonic voltage component

\section{CONCLUSIONS}

Through the use of de-embedding and active harmonic load pull, the demonstration of a Si LDMOS device in class F operation has been shown to produce record levels of efficiency at S-band frequencies. De-embedding to the current generator plane was essential to allowed for waveform engineering to be undertaken at the current generator plane. This also allows for direct comparison of the device performance at the different frequencies of operation. This has been confirmed by comparing a similar investigation conducted at $0.9 \mathrm{GHz}$ and good agreement was found in terms of performance and in the current and voltage waveforms.

\section{ACKNOWLEDGEMENTS}

The authors wish to acknowledge Freescale Semiconductor, Tempe, AZ for supplying the devices and the MET model.

\section{REFERENCES}

[1] S. C. Cripps, RF power amplifiers for wireless communications, $2^{\text {nd }}$ Ed. Boston, MA, Artech House, 2006.

[2] C. Roff et al, "Design approach for realization of very high efficiency power amplifiers," IEEEMTT-S Int. Microwave Symp. Digest, pp. 143-146, June 2007.

[3] C. Roff et al, "Optimising AlGaN/GaN HFET designs for high efficiency," Proceedings of the $2^{\text {nd }}$ European Microwave Integrated Circuits Conference, pp. 165-168, Oct 2007.

[4] A. Sheikh, et al, "The impact of system impedance on the characterization of high power devices," Proceedings of the $37^{\text {th }}$ European Microwave Conference, pp. 949-952, Oct 2007.

[5] J. Benedikt et al, "High power time domain measurement system with active harmonic load-pull for high efficiency base station amplifier design", IEEE MTT-S Int. Microwave Symp. Digest, pp. 1459-1462, June 2002.

[6] Young Yun et al,, "Efficiency Comparison Between Highly Efficient Class-F and Inverse Class-F Power Amplifiers" IEEE Microwave Magazine, pp. 100-110, June 2007.

[7] R. Gaddi, et al., "Direct extraction of LDMOS small signal parameters using off-state Measurements", IEE Electronic Letters, vol. 36, pp. 1964-1966, November 2000.

[8] W.R. Curtice, et al., "A new dynamic electro-thermal nonlinear model for silicon LDMOS FETs", IEEE Int. Microwave Symp. Dig., Vol 1, 1999, pp. 419-422.

[9] J.D. Rhodes, "Output universality in maximum efficiency linear power amplifiers," International Journal of Circuit Theory and Applications, vol. 31, pp.385-405, 2003. 